\title{
Complementary
}

Copyright (C) All rights are reserved by M Ghnaimat

\section{Management of Recurrent Anterior Shoulder Dislocation with Glenoid Bone Deficiency}

\author{
Malek Ghnaimat ${ }^{1 *}$, Mohannad Alodat ${ }^{1}$, Mohammad Aljazzazi $^{1}$, Muthana Alyamani ${ }^{1}$ and Raid Alsmadi ${ }^{2}$ \\ ${ }^{1}$ Department of Orthopedic, Royal Medical Services, (RMS), Jordan \\ ${ }^{2}$ Department of Anesthesia, Royal Medical Services, (RMS), Jordan
}

*Corresponding author : M Ghnaimat, Department of Orthopedic, Royal Medical Services, (RMS), Amman-Jordan.

Received Date: July 13, 2019

Published Date: July 31, 2019

\section{Abstract}

Objective: To assess the results of patients treated for recurrent anterior shoulder dislocation with glenoid bone deficiency by using the Latarjet procedure.

Methods: This is a retrospective study done in the period between April 2014 till February 2016 at the Jordanian Royal Medical Services (JRMS). Thirty patients with recurrent traumatic anterior shoulder dislocation who underwent surgical treatment with modified Latarjet technique were included in the study. Patients were questioned about satisfaction, range of motion and its effect on daily life and were examined for stability and range of motion and complications if present.

Result: 21 patients $96.6 \%$ were satisfied of the surgery with no experience of redislocation. One patient 3.3\% was not satisfied because of axillary nerve injury. Four patients $13.3 \%$ had a limitation of external rotation ranging from 5-15 degree. All patients returned to their level of activity at three months except the one with nerve injury fortunately he was improving.

Conclusion: The Modified Latarjet procedure is an effective technique for the treatment of recurrent anterior shoulder dislocation particularly associated glenoid bone loss.

Keywords: Latarjet procedure; Hillsachs lesion; Glenoid deficiecny shoulder dislocation

\section{Introduction}

Generally, it is well known that the incidence of shoulder instability is about $2 \%$. The most common complication of shoulder dislocation is recurrent dislocation owing to labral detachment or bony pathology in the glenoid and humeral head, which is known as hillsachs lesion. After the first time of anterior dislocation; glenohumeral deficiency either humeral head and glenoid defect or both has been found in up to $70 \%$ of the patients.

As previously mentioned, that Glenoid bone loss is commonly observed in recurrent anterior shoulder dislocation and varies in its extent [1]. In 2000, Burkhart and De Beer reported a recurrence rate of $4 \%$ following arthroscopic Bankart repair for anterior instability in patients without severe bone deficiency, whereas the rate of recurrence was $67 \%$ in the presence of severe glenoid deficiency [2]. In 1954, Latarjet described a coracoid transfer technique fixed to the anteroinferior margin of the glenoid with screws helpful in cases of glenoid bone loss [3-
6]. The working mechanism was explained by Patte: The effect of conjoint tendon when the arm is in abduction and external rotation acting as a sling that works on anteroinferior capsule and inferior of subscapularis muscle, repair of the coracoacromial ligament (CAL) with the capsule and the bony effect of increasing the glenoid anteroposterior diameter. The objective of this study is to assess the results of patients treated well using modified Laterjet Procedure.

\section{Methods}

A retrospective analysis of 30 male patients aged 20 to 28 years with recurrent traumatic anterior shoulder dislocation who underwent surgical treatment with modified Laterjet Technique was carried out. Ethical Committee Approval was obtained.

This study was conducted at Prince Hashim Military Hospital (PHMH) within the period of April 2014 to February 2016. Patients included in this study were all male and not previously operated. 
Also, they had plain X-rays and CT scan (figure 1) for their shoulders and reported to have Anterior Glenoid Deficiency. The operations were carried out for the patients by the team of (PHMH), and they were followed up in the clinic at periods between three and five months. During their clinic visit they were asked about satisfaction, range of motion and its effect on daily life activities, also the doctor was aware of asking them about their ability of returning back to the pre traumatic level of sports. In addition to that they were examined for the stability, range of motion, and any complications (Figure 1).

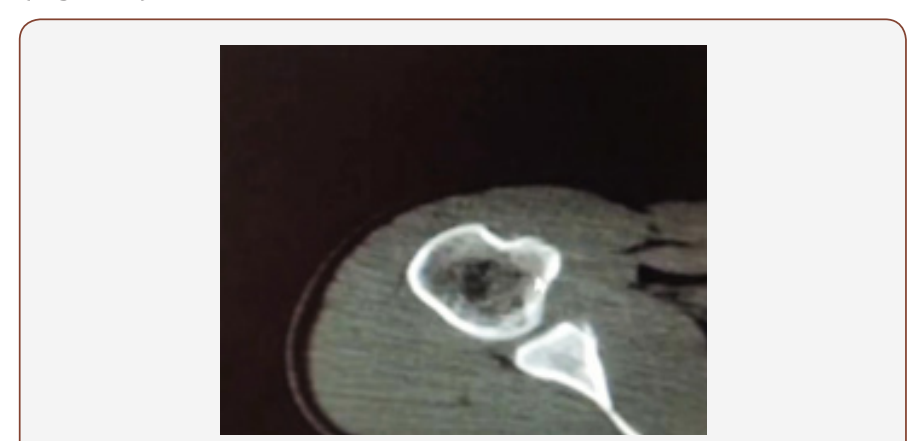

Figure 1: CT scan of left shoulder with glenoid deficiency and hillsachs lesion.

The modified Latarjet surgical procedure was via a deltopectoral approach with the cephalic vein and deltoid muscle retracted laterally. The arm was placed in external rotation with some abduction to expose the coracoacromial ligament CAL, which was incised $1 \mathrm{~cm}$ from its coracoid attachment. The coracohumeral ligament, found beneath the CAL, was then released. In addition to that, the pectoralis minor was released from the coracoids. Then the coracoid osteotomy was made from medial to lateral. In order to decorticate the inferior coracoid surface and have a wide cancellous

Table 2: Findings of the study. surface for graft healing an oscillating saw was used. Two drill holes were made using a $3.2 \mathrm{~mm}$ drill and about $1 \mathrm{~cm}$ apart. The location of the subscapularis split was at the junction of the superior two thirds and the inferior one third, the scissors were pushed between the fibers as far as the capsule, then opened perpendicular to the direction of the muscle fibers. A 1 to $2 \mathrm{~cm}$ vertical incision in the capsule was made at the level of the joint line, allowing placement of a retractor into the glenohumeral joint. The anteroinferior labrum and periosteum were incised and decortication of the anteroinferior surface of the glenoid was also done. Using the 3.2 $\mathrm{mm}$ drill, the first hole was created at the 5 o'clock position in the glenoid, then the second screw was placed. Later CAL was sutured with the capsule in external rotation.

\section{Result}

Patients' demographic data is shown in (Table I).

Table 1: Demographic data of patients.

\begin{tabular}{|c|c|c|c|c|c|}
\hline $\begin{array}{c}\text { Number } \\
\text { of } \\
\text { Patients }\end{array}$ & $\begin{array}{c}\text { Age } \\
\text { (Year) }\end{array}$ & $\begin{array}{c}\text { Gender } \\
\text { (M:F) }\end{array}$ & $\begin{array}{c}\text { (Right } \\
\text { Shoulder: } \\
\text { Left } \\
\text { Shoulder) }\end{array}$ & $\begin{array}{c}\text { Average } \\
\text { Dislocation } \\
\text { Time (Year) }\end{array}$ & $\begin{array}{c}\text { Average } \\
\text { Surgery } \\
\text { Time } \\
\text { (Year) }\end{array}$ \\
\hline 30 & $\begin{array}{c}24.3 \pm \\
(2.5)\end{array}$ & $30: 0$ & $16: 14$ & $1 \pm(2.6)$ & $\begin{array}{c}1.3 \pm(4.3) \\
\text { post first } \\
\text { dislocation }\end{array}$ \\
\hline
\end{tabular}

After retrospective analysis, the obtained results are shown in (Table 2). Table I represents the results of our study which was performed on thirty patients with average age of 24 years, all of them were males and almost half of which right shoulder and the other half left shoulder, and it shows that the time period of dislocations is about 1year up to 5 years. Also, it shows that the time of surgery post the first-time dislocation was about 1 to 4 years (Table 2).

\begin{tabular}{|c|c|c|c|c|c|}
\hline General Assessment & \multicolumn{4}{|c|}{ Clinical Assessment } \\
\hline $\begin{array}{c}\text { Satisfied with the Surgery } \\
\text { with no Experience of } \\
\text { Islocation }\end{array}$ & $\begin{array}{c}\text { Not Satisfied because } \\
\text { of Axillary Nerve } \\
\text { Injury }\end{array}$ & $\begin{array}{c}\text { Limitation of External } \\
\text { Rotation Ranging from } \\
\text { 5-15 Degree }\end{array}$ & $\begin{array}{c}\text { Axillary Nerve } \\
\text { Neuropraxia }\end{array}$ & $\begin{array}{c}\text { Radiolocation } \\
\text { Cases }\end{array}$ & $\begin{array}{c}\text { Limitation of } \\
\text { External Location }\end{array}$ \\
\hline $29(96.61 \%)$ & $1(3.3 \%)$ & $4(13.3 \%)$ & $1(3.3 \%)$ & -- & $4(13.3 \%)$ \\
\hline
\end{tabular}

All patients were returned to their previous levels of activity after three months, except the one with axillary nerve injury who is recovering, regained sensation at the glenoid region.

\section{Discussion}

Laterjet operation was first described by the French surgeon Dr. Michel Latarject in 1954 and it is considered as the best procedure for treating recurrent shoulder dislocations caused by bone loss or a fracture of the glenoide [2,3]. A lot of modifications of laterjet procedure were reported such as splitting of the subscapularis muscle and repair of CAL to capsule in external rotation led to excellent results in form of stability and excellent range of motion and early return to activities [4-6].

The study of Aurich et al on sixty patients undergoing modified latarjet showed that accompanied CAL reconstruction improved the shoulder function without redislocation [6], which was in agreement with what was obtained in the current study in the case of CAL repair to the capsule in external rotation.

A lot of intraoperative complications of Laterjet could appear such as neurovascular injuries and graft fracture, which could be avoided by the well understanding of anatomy and meticulous surgery in addition to the complications of intermediate term as nonunion and screw breakage, long term arthritis, and recurrent instability [7].

In the present study just one case $3.3 \%$ with axillary nerve neuropraxia was obtained which is better than what was previously obtained in Shah et al where 78 patients underwent open Latarjet procedures and 5 cases of them $6.5 \%$ had nerve injuries reported as Axillary,.Radial and.Musculocutaneous nerves [8]. This study also showed no instability or recurrent dislocation, while $5.8 \%$ and $8 \%$ recurrent dislocation were previously reported by Mizuno et al and 
Anab et al respectively $[3,8]$ Interestingly in this study the surgery yielded 4 patients of the thirty with limited external rotation and two of them were with about 5 degrees which was not affecting their daily life and sport level. On the other hand, a decrease in the external rotation in 90degree abduction and forward flexin of 5degrees was previously reported by Cresswell et al. [4] While Bradley et al reported 98\% success with no loss of external rotation or dislocation with patient selection and systematic surgical technique $[1,11]$.

Regarding to the occurrence of Arthritis in our study the follow up period is considered short to check for its appearance. Since in Mizuno et al 20 years follow up period for arthritis was reported, and it was found that $23 \%$ has developed mild Arthritis and usually developed in high demand activities and elder ages $[3,12,13]$.

\section{Conclusion}

The result of this study showed that the modified Laterjet procedure is considered as an excellent technique for treating recurrent anterior shoulder dislocation especially with glenoide bone deficiency. Most of the reported complications could be avoided with proper patient selection and meticulous surgical technique.

\section{Acknowledgement}

None.

\section{Conflicts of Interest}

No Conflicts of interest.

\section{References}

1. T Bradley Edwards, Gilles Walch (2012) The latarjet procedure for recurrent anterior shoulder instability: Rationale and Technique. Operative Techniques in Sport Medicine and Arthroscopy 20(1): 57-64.
2. Burkhart SS, De Beer JF (2000) Traumatic glenohumeral bone defects and their relationship to failure of arthroscopic Bankart repairs: Significance of the inverted-pear glenoid and the humeral engaging HillSachs lesion. Arthroscopy 16(7): 677-694.

3. Mizuno N, Denard PJ, Melis B, Walch G (2014) Long-term results of the Latarjet procedure for anterior instability of the shoulder. Shoulder Elbow Surg 23(11):1691-1699.

4. Cresswell T, Pritchard MG, De Beer JF (2005) Modified Latarjet procedure-technique and outcome. J Bone Joint Surg Br 87(2): 160.

5. Ebrahimzadeh MH, Moradi A, Zarei AR (2015) Minimally invasive modified Latajet in patients with traumatic anterior shoulder instability. Asian J Sports Med 6(1): 26-38.

6. Yamamoto N, Itoi E (2015) Osseous defects seen in patients with Anterior Shoulder Insatiability. Clin Orthop Surg 7(4): 425-429.

7. Colgate T, Watt CV, Joe De Beer J (2015) Evaluation of functional outcomes and complications following modified Latarjet reconstruction in athletes with anterior shoulder instability. Shoulder and Elbow 7(3): 168-173.

8. Aurich M, Gunther O, Hofmann, Gras F (2015) Reconstruction of the coracoacromial ligament during a modified Latarjet procedure: A case series. BMC Musculoskeletal disorders 16: 238.

9. Gupta A, Delaney R, Petkin K, Lafosse L (2015) Complications of the Latarjet procedure. Curr Rev Musculoskelet Med 8: 59-66.

10. Shah AA, Butler RB, Romanowski J, Warner J (2012) Short- term complications of the Laterjet procedure. The journal of bone and joint surgery 94(6): 495-501.

11. Denard PJ, Dai X, Burkhart SS (2015) Increasing preoperative dislocation and total time of dislocations affect surgical management of anterior shoulder instability. Int J Shoulder Surg 9(1): 1-5

12. Mc Laughlin RJ, Miniaci A, Jones MH (2015) Bony Versus Soft Tissue Reconstruction for Anterior Shoulder Instability: An expected value Decision analysis. Orthop J Sports Med 16: 3(12).

13. Da Silva, La da Costa lima AG, Kautsky RM, SantosPD, DoVal Sila G, et al. (2015) Evaluation of the results and complications of the Latarjet procedure for recurrent anterior dislocation of the shoulder. Rev bras ortop 50(6): 652-659. 